\title{
A Necessary Condition for existence of Lie Symmetries in Quasihomogeneous Systems of Ordinary Differential Equations
}

\author{
Y. Hirata ${ }^{\mathrm{a} *}$ and K. Imai ${ }^{\mathrm{b} \dagger}$ \\ aDepartment of Physics, Nagoya University, \\ Nagoya, 464-8602, Japan \\ ${ }^{b}$ Departments of Engineering, Daido Institute of Technology, \\ Nagoya, 457-8530, Japan
}

\begin{abstract}
Lie symmetries for ordinary differential equations are studied. In systems of ordinary differential equations, there do not always exist non-trivial Lie symmetries around equilibrium points. We present a necessary condition for existence of Lie symmetries analytic in the neighbourhood of an equilibrium point. In addition, this result can be applied to a necessary condition for existence of a Lie symmetry in quasihomogeneous systems of ordinary differential equations. With the help of our main theorem, it is proved that several systems do not possess any analytic Lie symmetries.
\end{abstract}

\section{Introduction}

A Lie symmetry is defined as a vector field which infinitesimally transforms a solution of a considered differential equation to, in general, another solution. Existence of Lie symmetries of differential equations gives us a lot of

\footnotetext{
${ }^{*}$ E-mail: yhirata@allegro.phys.nagoya-u.ac.jp
}

${ }^{\dagger}$ E-mail: kimai@daido-it.ac.jp 
information on dynamics, e.g., existence of similarity solutions, phase space structure, solvability of the system, and so on 1, 2].

For a system of ordinary differential equations, a Lie symmetry can be regarded as a vector field which commute with the vector field accompanying with the ordinary differential equations. However, in general, it is difficult to search Lie symmetries.

Hereby, it is known that existence or non-existence of first integrals has relevant to Kowalevskaya exponents, first named by Yoshida. Yoshida argued a necessary condition for existence of first integrals by using Kowalevskaya exponents[3]. Later Yoshida's theorem was extended to a necessary condition for existence of invariant tensor fields with general orders by Kozlov 4 . Although above two theorems seem powerful, they have an important weak point. Their theorems gives some conditions for Kowalevskaya exponents under existence of a "non-degenerate" first integral (or invariant tensor field). Therefore even if the conditions are not satisfied, one can claim only nonexistence of "non-degenerate" ones.

Recently Furta[5] and Goriely [6] independently overcame this weak point although the assertion is a little weak. Because this method does not suppose non-degeneracy of first integrals, one can use the method for proofs of nonexistence of first integrals. In this paper we show a necessary condition for existence of analytic Lie symmetries by using Furta's method.

This paper is outlined as follows. Local Lie symmetries always exist around regular points of a system of ordinary differential equations. However in the neighbourhood of an equilibrium point, there are not necessarily nontrivial Lie symmetries. In the next section we give a necessary condition for existence of Lie symmetries around an equilibrium point. We devote Section 3 to an introduction of quasihomogeneous (similarity invariant or weighted homogeneous) systems and Lie symmetries, which are main subjects in this paper. We give our main theorem and its proof in Section 4. Section 5 is devoted to extension of the previous section to semi-quasihomogeneous systems, which is defined in the section. A concrete example is given in Section 6. We conclude this paper in Section 7 . 


\section{Ordinary differential equations and Lie sym- metries}

In this section, we present a basic lemma on existence of Lie symmetries in a system of analytic ordinary differential equations. It can be regarded as a necessary condition for existence of local Lie symmetries around an equilibrium point of a system of analytic ordinary differential equations.

Assume the system

$$
\dot{x}=g(x), \quad x \in \mathbf{C}^{n}
$$

is analytic in the neighbourhood of the origin $x=0$. We also write the system (1) as

$$
\dot{x}^{i}=g^{i}(x), \quad i=1, \ldots n
$$

by using the $i$-th component of $x$ and $g$. Because we are interested in Lie symmetries around an equilibrium point, we set $g(0)=0$ without loss of generality.

We define Lie symmetries of the system (11) (or equivalently (2)). Let $X_{g}$ be the vector field accompanying with the system (11), that is,

$$
X_{g}=\sum_{i=1}^{n} g^{i}(x) \frac{\partial}{\partial x^{i}}
$$

Definition 1 (Lie symmetries). A vector field

$$
X_{\phi}=\sum_{i=1}^{n} \phi^{i}(x) \frac{\partial}{\partial x^{i}}
$$

is called a Lie symmetry of the system (11) if the vector field (3) commutes with $X_{g}$ in the sense of the Lie bracket, that is,

$$
\left[X_{g}, X_{\phi}\right]:=X_{g} X_{\phi}-X_{\phi} X_{g}=0 \text {. }
$$

Remark 1. In general, one has to set Lie symmetries in the form of

$$
X=\tau(x, t) \frac{\partial}{\partial t}+\sum_{i=1}^{n} \phi^{i}(x, t) \frac{\partial}{\partial x^{i}} .
$$

However, in this paper, we restrict ourselves to autonomous Lie symmetries in the form of the expression (3). 
We say an analytic Lie symmetry or a polynomial Lie symmetry if all the components of the vector field $\phi^{i}(x)$ are analytic or polynomial, respectively. In addition, let us define order of Lie symmetries.

Definition 2 (Order of vector fields). An analytic vector field (or Lie symmetry) $X_{\phi}=\sum \phi^{i}(x) \partial / \partial x^{i}$ is of order $k$ if the Taylor expansion of the coefficient vector $\phi(x)={ }^{t}\left(\phi^{1}(x), \ldots, \phi^{n}(x)\right)$ starts from $k$-th order terms.

The following statement holds on an analytic Lie symmetry around an equilibrium point.

Lemma 1. Set $\partial g / \partial x(0)=A$, and $\lambda_{1}, \ldots, \lambda_{n}$ are eigenvalues of $A$. Suppose $\operatorname{det} A \neq 0$ and $A$ is diagonalizable. If the system (1) possesses an analytic Lie symmetry of order $k$ in the neighbourhood of the origin, then for some $j \in\{1, \ldots, n\}$, a resonance condition

$$
\sum_{i=1}^{n} k_{i} \lambda_{i}=\lambda_{j}, \quad k_{i} \in \mathbf{N} \cup\{0\}, \quad \sum_{i=1}^{n} k_{i}=k
$$

holds.

Remark 2. The assumption $\operatorname{det} A \neq 0$ is not necessary. In fact, if $\operatorname{det} A=$ 0 , the set $\lambda_{1}, \ldots, \lambda_{n}$ always satisfies at least one resonance relation of the type (4).

Remark 3. The resonance relation (4) possesses a trivial solution $k_{i}=\delta_{i j}$. This corresponds with the fact that any vector field trivially commute with itself. Therefore the contrapositive statement of the lemma does not tell nonexistence of Lie symmetries of order 1.

Proof. Let $X_{\phi}$ be an analytic Lie symmetry of order $k$. Therefore the relation $\left[X_{g}, X_{\phi}\right]=0$ holds. $A$ has already been diagonalized and hence $A=\operatorname{diag}\left(\lambda_{1}, \ldots, \lambda_{n}\right)$.

From the analyticity of $X_{\phi}$, we can expand $X_{\phi}$ into the power series with respect to $x$ as

$$
X_{\phi}=X_{\phi_{k}}+X_{\phi_{k+1}}+\ldots
$$


where

$$
X_{\phi_{m}}=\sum_{j=1}^{n} \phi_{l}^{j}(x) \frac{\partial}{\partial x^{j}}, \quad m=k, k+1, \ldots
$$

and $\phi_{m}^{i}$ are homogeneous polynomials of order $m$.

Since the lowest order terms of $\left[X_{g}, X_{\phi}\right]$ must be zero, we obtain the relation

$$
\left[X_{A x}, X_{\phi_{k}}\right]=0
$$

Let us explicitly write $\phi_{k}^{j}(x)$ as

$$
\phi_{k}^{j}(x)=\sum_{k_{1}+\cdots+k_{n}=k} \phi_{k_{1} \ldots k_{n}}^{j}\left(x^{1}\right)^{k_{1}} \ldots\left(x^{n}\right)^{k_{n}} .
$$

Straightforward computation gives

$$
\sum_{j=1}^{n} \sum_{k_{1}+\cdots+k_{n}=k}\left(\sum_{i=1}^{n} \lambda_{i} k_{i}-\lambda_{j}\right) \phi_{k_{1} \ldots k_{n}}^{j}\left(x^{1}\right)^{k_{1}} \ldots\left(x^{n}\right)^{k_{n}} \frac{\partial}{\partial x_{j}}=0 .
$$

Since $X_{\phi}$ is of order $k$, at least one coefficient $\phi_{k_{1} \ldots k_{n}}^{j}$ is not zero. This implies the condition (田).

\section{Quasihomogeneous systems and Lie sym- metries}

Consider the system

$$
\dot{x}=f(x), \quad x \in \mathbf{C}^{n}
$$

where $f(x)$ is analytic in the neighbourhood of the origin. We define quasihomogeneous systems, which is also called similarity invariant systems or weighted homogeneous systems.

Definition 3 (Quasihomogeneous systems [3]). The system (5) is called a quasihomogeneous system if the system is invariant under the transformation

$$
t \mapsto \alpha^{-1} t, \quad x^{i} \mapsto \alpha^{g_{i}} x^{i}, \quad g_{i} \in \mathbf{Q}, \quad i=1, \ldots, n
$$




$$
\begin{aligned}
& \text { for } \forall \alpha \in \mathbf{R}^{+} \text {, i.e., } f^{i}(x), i=1, \ldots, n \text { satisfy } \\
& \qquad f^{i}\left(\alpha^{g_{1}} x^{1}, \ldots, \alpha^{g_{n}} x^{n}\right)=\alpha^{g_{i}+1} f^{i}\left(x^{1}, \ldots, x^{n}\right) .
\end{aligned}
$$

Hereafter we refer $g_{1}, \ldots, g_{n}$ as the weight exponents.

Remark 4. By defining $G=\operatorname{diag}\left(g_{1}, \ldots, g_{n}\right)$ and $\alpha^{G}=\operatorname{diag}\left(\alpha^{g_{1}}, \ldots, \alpha^{g_{n}}\right)$, the expression (7) can be rewritten as

$$
f^{i}\left(\alpha^{G} x\right)=\alpha^{g_{i}+1} f^{i}(x), \quad i=1, \ldots, n .
$$

Moreover, since $f={ }^{t}\left(f^{1}, \ldots, f^{n}\right)$, we can simply write the expression (8) as

$$
f\left(\alpha^{G} x\right)=\alpha^{G+E} f(x)
$$

where $E$ is the $n$-dimensional unit matrix.

Choose $l$ be the smallest positive integer such that all the quantities $l g_{1}, \ldots, l g_{n}$ are integers. Then we call the system (5) a quasihomogeneous system of ramification degree $l$.

Let us define degrees of quasihomogeneous functions and quasihomogeneous Lie symmetries.

Definition 4 (Quasihomogeneous functions). A function $F(x)$ is called a quasihomogeneous function of degree $M$ if the relation

$$
F\left(\alpha^{G} x\right)=\alpha^{M / l} F(x)
$$

holds.

Proposition 1. An analytic quasihomogeneous function has an integer degree.

Proof. Set $S=l G$ and $\alpha^{1 / l}=\mu$. Hence the expression (10) can be rewritten as

$$
F\left(\mu^{S} x\right)=\mu^{M} F(x) .
$$

The 1.h.s. of the expression (11) can be expanded into the Laurent series with respect to $\mu$ from the analyticity of $F$. This implies $M$ must be an integer. 
Definition 5 (Quasihomogeneous Lie symmetries). We call a Lie symmetry $X_{\phi}$ a quasihomogeneous Lie symmetry of degree $M$ if all the coefficients $\phi^{i}(x), i=1, \ldots, n$ are quasihomogeneous functions of degree $M+g_{i} l$.

Remark 5. If $X_{\phi}$ is a Lie symmetry of degree $M$, the following relation holds:

$$
\left.X_{\phi}\right|_{\alpha^{G} x}=\alpha^{M / l} X_{\phi} .
$$

For quasihomogeneous systems, it is sufficient to consider quasihomogeneous Lie symmetries. In fact, every analytic Lie symmetry $X_{\phi}$ splits into the power series of $\alpha$ with quasihomogeneous vector field coefficients by the transformation (6), i.e.,

$$
\left.X_{\phi}\right|_{\alpha^{G} x}=\sum_{M} \alpha^{M / l} X_{\phi_{M}},
$$

where $X_{\phi_{M}}$ are quasihomogeneous vector fields of degree $M$. Since $\alpha$ is arbitrary, each of $X_{\phi_{M}}$ must be a Lie symmetry. Hence we restrict ourselves to quasihomogeneous Lie symmetries.

Quasihomogeneous systems (5) possess a particular solution in the form

$$
x(t)=c t^{-G}
$$

where $c \neq 0$ is a solution vector of the equation

$$
f(c)+G c=0 .
$$

Consider variation of the system (5) around the particular solution (12). Let us make the following change of variables $x \mapsto u$ such that

$$
x=t^{-G}(c+u) .
$$

Substituting the expression (14) into the equation (5) and using the relation (9), we have

$$
-G t^{-G-E}(c+u)+t^{-G} \dot{u}=t^{-G-E} f(c+u) .
$$

Introducing the new independent variable $\tau=\log t$, the equation (15) can be expressed as an autonomous system

$$
u^{\prime}=G c+G u+f(c+u),
$$


where ${ }^{\prime}=d / d \tau$. Moreover, from the analyticity of $f$, one can expand $f$ around $x=c$ and has

$$
f(c+u)=f(c)+\frac{\partial f}{\partial x}(c) u+\tilde{f}(u)
$$

where $\tilde{f}$ stands for higher order terms. Substituting the equations (17) and (13) into the equation (16), we have

$$
u^{\prime}=\left(\frac{\partial f}{\partial x}(c)+G\right) u+\tilde{f}(u)
$$

The quantity in the bracket of the r.h.s. of the equation (18) is called the Kowalevskaya matrix.

Definition 6 (Kowalevskaya matrix and exponents[3]). The $n \times n m a$ trix

$$
K=\frac{\partial f}{\partial x}(c)+G
$$

is called the Kowalevskaya matrix. Moreover the eigenvalues of $K$ is called the Kowalevskaya exponents.

Thus the quasihomogeneous system (5) can be expressed as

$$
u^{\prime}=K u+\tilde{f}(u)
$$

around the particular solution (14). In this paper, we assume that Kowalevskaya matrices are diagonalizable.

Lemma 2 ([3, 5]). $\lambda=-1$ is an eigenvalue of a Kowalevskaya matrix for an autonomous system.

Proof. We show that $\lambda=-1$ is an eigenvalue of $K$ defined as the expression (19) and $q=G c$ is an eigenvector belonging to $\lambda=-1$. First by differentiating the equation (9) with respect to $\alpha$ and substituting $\alpha=1$ and $x=c$, one obtains

$$
\frac{\partial f}{\partial x}(c) G c=(G+E) f(c)
$$


On the other hand,

$$
K q=K G c=\left(G+\frac{\partial f}{\partial x}(c)\right) G c .
$$

By substituting the equation (21) into the equation (22), we have

$$
K q=G^{2} c+(G+E) f(c)=G(G c+f(c))+f(c) .
$$

Furthermore, since $G c+f(c)=0$,

$$
K q=f(c)=-G c=-q
$$

The lemma is proved.

\section{Main Theorem}

Now we give our main theorem:

Theorem 1 (Main Theorem). Let the Kowalevskaya matrix $K$ of the quasihomogeneous system (5) be diagonalizable, and $\lambda_{1}, \ldots, \lambda_{n}$ be the Kowalevskaya exponents. Set $\lambda_{1}=-1$ and put $\lambda_{0}=1 / l$, where $l$ is the ramification degree of the system. If the quasihomogeneous system has an analytic Lie symmetry of degree $M(\geq 0)$, then, for some $j \in\{0,1, \ldots, n\}$, the resonance condition

$$
\sum_{i=1}^{n} k_{i} \lambda_{i}=l \lambda_{j}, \quad k_{i} \in \mathbf{N} \cup\{0\}, \quad k_{1} \geq M
$$

holds.

Proof. Let the vector field

$$
X_{\phi}=\sum_{i=1}^{n} \phi^{i}(x) \frac{\partial}{\partial x^{i}}
$$

be a quasihomogeneous Lie symmetry of degree $M$. The quasihomogeneous system (5) is transformed into the autonomous system (20) by the transformation (14). Applying the transformation (14) to the Lie symmetry (24), we have

$$
X_{\phi}=t^{-M / l} \sum_{i=1}^{n} \phi^{i}(c+u) \frac{\partial}{\partial u^{i}}
$$


Putting $t^{-1 / l}=u^{0}$, we have Thus

$$
X_{\phi}=\left(u^{0}\right)^{M} \sum_{i=1}^{n} \phi^{i}(c+u) \frac{\partial}{\partial u^{i}},
$$

which is analytic with respect to $\left(u^{0}, u^{1}, \ldots, u^{n}\right)$. Then the new independent variable $u^{0}$ satisfies a linear ordinary differential equation

$$
\left(u^{0}\right)^{\prime}=-\frac{1}{l} u^{0} .
$$

A straightforward computation tells that the transformed vector field (25) is a Lie symmetry of the extended autonomous system

$$
\left\{\begin{aligned}
\left(u^{0}\right)^{\prime} & =-\frac{1}{l} u^{0} \\
u^{\prime} & =K u+\tilde{f}(u) .
\end{aligned}\right.
$$

Therefore, applying Lemma \&, we have a resonance relation

$$
-\frac{k_{0}}{l}+\sum_{i=1}^{n} k_{i} \lambda_{i}=\lambda_{j}, \quad k_{0}, \ldots, k_{n} \in \mathbf{N} \cup\{0\}, \quad k_{0}+\sum_{i=1}^{n} k_{n} \geq M,
$$

where $k_{0}=M$. Multiplying $l$ to the both hands of the first equation in the expression (26) and rewriting $k_{0}+l k_{1} \rightarrow k_{1}$ and $l k_{i} \rightarrow k_{i}, i=2, \ldots, n$, we obtain the expression (23). This completes the proof.

\section{Semi-quasihomogeneous systems}

We reconsider an $n$-dimensional autonomous system

$$
\dot{x}=f(x), \quad x \in \mathbf{C}^{n}
$$

which are analytic in the neighbourhood of the origin. Let the $j$-th component of $f$ be expanded into the Maclaurin series

$$
f^{j}(x)=\sum f_{k_{1} \ldots k_{n}}^{j}\left(x^{1}\right)^{k_{1}} \ldots\left(x^{n}\right)^{k_{n}} .
$$


Proposition 2 ([5]). If the system (27) is quasihomogeneous with respect to the weight exponents $g_{1}, \ldots, g_{n} \in \mathbf{Q}$, then all the terms in the expansion (28) satisfy the equation

$$
g_{1} k_{1}+\cdots+g_{n} k_{n}=g_{j}+1, \quad j=1, \ldots, n .
$$

Now let us define semi-quasihomogeneous systems.

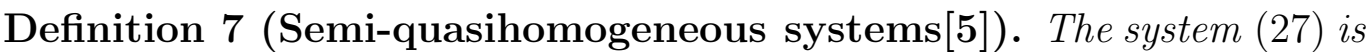
called a semi-quasihomogeneous system if it can be expressed as the form

$$
\dot{x}=f_{m}(x)+\hat{f}(x),
$$

where $f_{m}(x)$ defines a quasihomogeneous system of degree $m$ and all the terms in the expansion of $\hat{f}^{j}(x)$, which is the $j$-th component of $\hat{f}(x)$, satisfy either

$$
g_{1} k_{1}+\cdots+g_{n} k_{n}>g_{j}+1, \quad j=1, \ldots, n
$$

or

$$
g_{1} k_{1}+\cdots+g_{n} k_{n}<g_{j}+1, \quad j=1, \ldots, n .
$$

In addition, if the inequality (30) (resp. (31)) holds, we call the system (29) a positively (resp. negatively) semi-quasihomogeneous system.

Let us give a theorem for existence of Lie symmetries in semi-quasihomogeneous systems:

Theorem 2 (Lie symmetries for semi-quasihomogeneous systems). If a positively (resp. negatively) semi-quasihomogeneous system (29) possesses an analytic (resp. polynomial) Lie symmetry, then the truncated system

$$
\dot{x}=f_{m}(x)
$$

has an analytic (resp. polynomial) Lie symmetry.

Proof. Applying the scale transformation (6) to the system (29), we have

$$
\dot{x}=f_{m}(x)+\hat{f}(x, \mu),
$$

where $\hat{f}(x, \mu)$ is a formal Taylor series with respect to $\mu(r e s p .1 / \mu)$ without constant terms. 
On the other hand, an analytic (resp. polynomial) Lie symmetry $X_{\phi}$ of the system (29) is transformed into the form $\mu^{M}\left(X_{M}+\mu X_{M+1}+\mu^{2} X_{M+2}+\right.$ $\ldots)\left(\right.$ resp. $\left.\mu^{M}\left(X_{M}+\frac{1}{\mu} X_{M-1}+\frac{1}{\mu^{2}} X_{M-2}+\cdots+\frac{1}{\mu^{N}} X_{M-N}\right)\right)$ for some $M$, where $X_{i}$ are quasihomogeneous vector fields of degree $i$. Hence

$$
\begin{gathered}
X_{M}+\mu X_{M+1}+\mu^{2} X_{M+2}+\ldots \\
\left(\text { resp. } X_{M}+\frac{1}{\mu} X_{M-1}+\frac{1}{\mu^{2}} X_{M-2}+\cdots+\frac{1}{\mu^{N}} X_{M-N}\right)
\end{gathered}
$$

is an analytic (resp. polynomial) Lie symmetry of the system (33).

The system (33) approaches the truncated system (32) as $\mu \rightarrow 0$ (resp. $\mu \rightarrow \infty)$. On the other hand, the Lie symmetry (34) (resp. (35)) approaches $X_{M}$ simultaneously. Thus the truncated system ( $\overline{32}$ ) possesses a Lie symme$\operatorname{try} X_{M}$.

\section{Example}

Consider the 2-dimensional quadratic systems

$$
\begin{aligned}
& \dot{x}^{1}=\left(x^{1}\right)^{2}+x^{1} x^{2} \\
& \dot{x}^{2}=a x^{1} x^{2}+\left(x^{2}\right)^{2}
\end{aligned}
$$

with a parameter $a$, which can be regarded as particular cases of the LotkaVolterra system.

The weight exponents of the system is $g_{1}=g_{2}=1$ and hence the ramification degree $l=1$. The vector $c$ is easily computed as

$$
c= \begin{cases}{ }^{t}\left(c_{1},-c_{1}-1\right), c_{1} \in \mathbf{C}, & a=1, \\ { }^{t}(-1,0),{ }^{t}(0,-1), & a \neq 1 .\end{cases}
$$

We get the first Kowalevskaya exponent $\lambda_{1}=-1$. The other Kowalevskaya exponent $\lambda_{2}=0$ or $1-a$.

We first consider the case $\lambda_{2}=0$. The resonance relation (23) is written as

$$
-k_{1}=-1, \quad k_{1} \geq 0, \quad k_{1} \geq M
$$

and

$$
-k_{1}=0, \quad k_{1} \geq 0, \quad k_{1} \geq M
$$


respectively. The equations (37) and (38) implies $k_{1}=0,1$, and hence $M \leq 1$. In this example $M \geq-1$, because analytic quasihomogeneous Lie symmetries with constant coefficients have degree -1 . Thus we check the case $M=$ $-1,0,1$. Straightforward computation gives the only case $a=1$ with nontrivial analytic Lie symmetries

$$
\begin{gathered}
X_{1}=x^{1} \frac{\partial}{\partial x^{1}}-x^{1} \frac{\partial}{\partial x^{2}}, \\
X_{2}=x^{2} \frac{\partial}{\partial x^{1}}-x^{2} \frac{\partial}{\partial x^{2}} \\
X_{3}=\left(x^{1}\right)^{2} \frac{\partial}{\partial x^{1}}+x^{1} x^{2} \frac{\partial}{\partial x^{2}},
\end{gathered}
$$

and

$$
X_{4}=x^{1} x^{2} \frac{\partial}{\partial x^{1}}+\left(x^{2}\right)^{2} \frac{\partial}{\partial x^{2}} .
$$

In other words, when $a \neq 1$, there are no analytic Lie symmetries. Incidentally, although we have the other second Kowalevskaya exponent $\lambda_{2}=1-a$ for $a \neq 1$, it is obvious that there exist no other analytic Lie symmetries.

The 4-dimensional Lie algebra $\mathcal{L}_{4}$ spanned by $\left\{X_{1}, X_{2}, X_{3}, X_{4}\right\}$ is solvable and therefore the system (36) can be integrated by using $\mathcal{L}_{4}$. The commutator table is given in Table 1 .

\begin{tabular}{r|rrrr} 
& $X_{1}$ & $X_{2}$ & $X_{3}$ & $X_{4}$ \\
\hline$X_{1}$ & 0 & $-X_{1}-X_{2}$ & $X_{3}$ & $-X_{3}$ \\
$X_{2}$ & $X_{1}+X_{2}$ & 0 & $X_{4}$ & $-X_{4}$ \\
$X_{3}$ & $-X_{3}$ & $-X_{4}$ & 0 & 0 \\
$X_{4}$ & $X_{3}$ & $X_{4}$ & 0 & 0
\end{tabular}

Table 1: The commutator table of 4-dimensional Lie algebra $\mathcal{L}_{4}$. One can take a sequence of sub-algebra $\mathcal{L}_{4} \supset \mathcal{L}_{3} \supset \mathcal{L}_{2}$, where $\mathcal{L}_{3}$ and $\mathcal{L}_{2}$ are subalgebras spanned by $\left\{X_{2}, X_{3}, X_{4}\right\}$ (or $\left.\left\{X_{1}, X_{3}, X_{4}\right\}\right)$ and $\left\{X_{3}, X_{4}\right\}$, respectively. Hence this Lie algebra is solvable.

Furthermore, by applying Theorem 2, the 2-dimensional Lotka-Volterra systems

$$
\begin{aligned}
& \dot{x}^{1}=a_{11} x^{1}+a_{12} x^{2}+\left(x^{1}\right)^{2}+x^{1} x^{2}, \\
& \dot{x}^{2}=a_{21} x^{1}+a_{22} x^{2}+a x^{1} x^{2}+\left(x^{2}\right)^{2}
\end{aligned}
$$


possess no polynomial Lie symmetries except the case $a=1$. Moreover the 2-dimensional replicator systems

$$
\begin{aligned}
& \dot{x}^{1}=\left(x^{1}\right)^{2}+x^{1} x^{2}-x^{1}\left(\left(x^{1}\right)^{2}+x^{1} x^{2}\right) \\
& \dot{x}^{2}=a x^{1} x^{2}+\left(x^{2}\right)^{2}-x^{2}\left(a x^{1} x^{2}+\left(x^{2}\right)^{2}\right)
\end{aligned}
$$

has no analytic Lie symmetries except the case $a=1$.

\section{Conclusion}

We give a necessary condition for existence of analytic Lie symmetries in quasihomogeneous systems of ordinary differential equations. If the resonance equation (23) possesses only finite solutions or no solutions, one can argue a question on non-existence of analytic Lie symmetries in the given systems. Indeed, we show non-existence of analytic Lie symmetries in the 2dimensional quadratic systems (36), which are particular cases of the LotkaVolterra system.

The system (36) with $a=1$ possesses the solvable Lie algebra $\mathcal{L}_{4}$. On the other hand, the system has a first integral $x_{1} / x_{2}$. Although our theorem is not lost this "solvable" case, Furta's theorem miss it because the first integral is not analytic in the neighbourhood of the origin. Thus our theorem surpasses Furta's theorem under such a situation.

The resonance equation (23) has either finite or infinite solutions. If the number of the solutions is finite, one can check the considered system possesses analytic Lie symmetries or not. On the other hand, if infinite solutions exist, it is difficult to argue non-existence of analytic Lie symmetries. The infinity of solutions is caused by solutions of the resonance equation in Furta's theorem[5]. In fact, if there exists an analytic first integral $\Phi$ with a positive degree, then the vector fields $\Phi X_{f}, \Phi^{2} X_{f}, \ldots$ are all analytic Lie symmetries. Thus our main theorem is consistent with Furta's theorem.

\section{Acknowledgements}

The authors would like to thank M. Ishii and T. Konishi for fruitful discussions. 


\section{References}

[1] P. J. Olver, Applications of Lie groups to differential equations, SpringerVerlag, New York, 1986.

[2] N. H. Ibragimov (eds.), CRC handbook of Lie group analysis of differential equations vol. 1-3, CRC, 1994-1996.

[3] H. Yoshida, Cele. Mech., 31 (1983) 363-379; 381-399.

[4] V. V. Kozlov, Math. Notes., 51 (1992) 138-142.

[5] S. D. Furta, Z. angew. Math. Phys., 47 (1996) 112-131.

[6] A. Goriely, J. Math. Phys., 37 (1996) 1871-1893; Regular and Chaotic Dynamics, 5 (2000) 3-15. 